\title{
On the Performance of the POSIX I/O Interface to PVFS
}

\author{
Murali Vilayannur ${ }^{\dagger} \quad$ Robert B. Ross ${ }^{\ddagger} \quad$ Philip H. Carns * \\ Rajeev Thakur ${ }^{\ddagger}$ Anand Sivasubramaniam ${ }^{\dagger} \quad$ Mahmut Kandemir $^{\dagger}$ \\ $\dagger$ Department of Computer Science and Engineering \\ Pennsylvania State University, University Park, PA 16802 \\ $\{$ vilayann, anand, kandemir\}@cse.psu.edu \\ $\ddagger$ Mathematics and Computer Science Division $\quad *$ Parallel Architecture Research Laboratory \\ Argonne National Laboratory, Argonne, IL 60439 Clemson University, Clemson, SC 29634 \\ \{rross, thakur\}@mcs.anl.gov \\ pcarns@parl.clemson.edu
}

\begin{abstract}
The ever-increasing gap in performance between CPU/memory technologies and the I/O subsystem (disks, I/O buses) in modern workstations has exacerbated the I/O bottlenecks inherent in applications that access large disk resident data sets. A common technique to alleviate the I/O bottlenecks on clusters of workstations, is the use of parallel file systems. One such parallel file system is the Parallel Virtual File System (PVFS), which is a freely available tool to achieve high-performance I/O on Linux-based clusters.

In this paper, we describe the performance and scalability of the UNIX I/O interface to PVFS. To illustrate the performance, we present experimental results using Bonnie ++, a commonly used file system benchmark to test file system throughput; a synthetic parallel I/O application for calculating aggregate read and write bandwidths; and a synthetic benchmark which calculates the time taken to untar the Linux kernel source tree to measure performance of a large number of small file operations. We obtained aggregate read and write bandwidths as high as 550 $\mathrm{MB} / \mathrm{s}$ with a Myrinet-based network and $160 \mathrm{MB} / \mathrm{s}$ with fast Ethernet.
\end{abstract}

\section{Introduction}

In recent years, the disparity between $\mathrm{I} / \mathrm{O}$ performance and CPU performance has led to $\mathrm{I} / \mathrm{O}$ bottlenecks in many applications that use large data sets. This gap is becoming more problematic as we move to multi-processor and cluster systems, where the compute power is multiplied by the number of processing units available. An opportunity for high-performance I/O exists on these platforms by harnessing the I/O subsystems on each node. The multiple CPUs and their memories can provide processing and primary storage parallelism, while the multiple disks can provide secondary storage parallelism for both data access and transfer. Parallel file systems exploit this feature to hide the I/O bottlenecks from the applications. While many commercial parallel file systems have been developed for supercomputers and parallel machines, such as PFS for the
Intel Paragon [1], and GPFS [13] for the IBM SP, and many academic endeavors, such as Galley [11], and PIOUS [10], many of these solutions are not available or not intended for production use on Linux-based clusters.

PVFS [5] is a freely available parallel file system that is intended both as a research tool in parallel I/O, and as a stable file system that can be used on production Linux clusters. The initial goals of PVFS were to provide data striping and file partitioning in a distributed environment and to provide an interface that is reasonably close to the standard UNIX I/O interface. The first prototype implementation was developed with the main goal of achieving high-performance I/O and was intended to be run on a 4-node Alpha cluster in 1994. In recent times, clusters of a thousand machines and more have become more common. Hence, the goals of PVFS have been redefined to achieve not only high performance but also scalability. In the past, PVFS has been optimized for bandwidthlimited parallel applications [5] that access the file system through the MPI-IO [8] and native library interface. PVFS has also been optimized for parallel applications with noncontiguous [6] access patterns. The UNIX I/O interface to PVFS is a fairly recent addition, which was intended more for convenience than for achieving high-performance I/O.

In this paper, we describe the key performance and scalability problems that hinder the use of standard UNIX I/O interfaces through PVFS for high-performance parallel $\mathrm{I} / \mathrm{O}$, and we present techniques that we have implemented to alleviate the following:

- File system overheads, such as the amount of buffer copying and the number of context switches between processes and client-side daemons.

- TCP/IP connection management overheads.

- Lack of aggregation in directory read operations.

We describe these problems and our proposed solutions in greater detail in subsequent sections. To illustrate the performance, we present experimental results obtained from the Bonnie++ [7] file system benchmark, a synthetic parallel I/O workload, and a synthetic workload which times the untar of the Linux kernel source tree. The experimental results presented in this paper are from a 16-node Intel Pentium-III Linux-based cluster of workstations. Each 


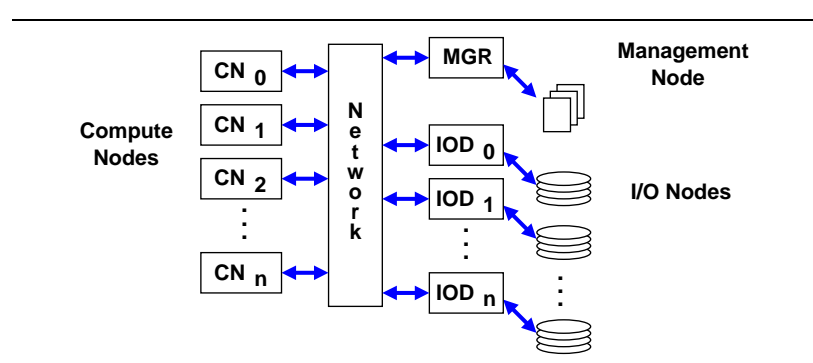

Figure 1. Overall system architecture

node on this cluster consists of dual $1 \mathrm{GHz}$ Intel PentiumIII microprocessor equipped with 1 GB RAM and two 30GB IDE-Maxtor hard drives. Each node also has two network interfaces, an Intel 82557 (Ethernet Pro100) fast Ethernet interface, and a Myrinet [4] (M3M-PCI64B-2 with $2 \mathrm{MB}$ of on-board RAM operating at $1.28 \mathrm{Gbps}$ ) interface. All the nodes are connected through fast Ethernet and Myrinet switches (three 8-port M2M-SW8 switches).

The rest of this paper is organized as follows. Section 2 outlines the design and implementation of PVFS, and Section 3 describes the performance and scalability bottlenecks and the enhancements proposed to alleviate them. Experimental results on a variety of benchmarks are presented in Section 4. Section 5 summarizes the contributions of this paper and discusses directions for further improvements.

\section{PVFS: System Architecture}

The goals of PVFS as a parallel file system are to provide high-speed access to file data for parallel applications. The main features of PVFS are that it provides a cluster-wide name space for clients to access their data files, enables users to control striping parameters when creating files, delivers scalable performance under concurrent reads/writes, and allows for legacy binaries to operate on PVFS volumes without having to be recompiled. PVFS is designed as a client-server system as shown in Figure 1 and described in greater detail below.

\subsection{PVFS: Servers}

PVFS uses two server components, both of which run as user-level daemons on one or more nodes of the cluster. One of these is a meta-data server (called MGR) to which requests for meta-data management (access rights, directories, file attributes and physical distribution of file data) are sent. In addition, there are several instances of a data server daemon (called IOD), one on each node of the cluster whose disk is being used to store data as part of the PVFS name space. There are well-defined protocol structures for exchanging information between the clients and the servers. For instance, when a client wishes to open a file, it communicates with the MGR daemon which provides it the necessary meta-data information (such as the location of IOD servers for this file, or stripe information) to do subsequent operations on the file. Subsequent reads and writes to this file do not interact with the MGR daemon and are handled directly by the IOD servers. This strategy is key to achieving scalable performance under concurrent reads and write requests from many clients and has been adopted by more recent parallel file system efforts.

\subsection{PVFS: Clients}

PVFS supports many different client APIs, such as:

- Native libpvfs API,

- Standard UNIX/POSIX API [2], and

- MPI-IO API $[8,15]$.

Applications written with the native library interface must be linked with libpvfs to read and write files on a PVFS file system. The native API has functions analogous to the POSIX API functions for contiguous reads and writes. In addition, it includes support for non-contiguous reads and writes with a single function call [6]. MPI-IO [8, 15] is an API for parallel I/O as part of the MPI-2 standard and contains features specifically designed for I/O parallelism and performance. The MPI-IO interface has been implemented on top of PVFS by using the ROMIO [15] implementation of MPI-IO. ROMIO is designed to be ported easily to new file systems by using an abstract-device interface[14]. PVFS also supports the standard UNIX I/O functions, such as open, close, read, and write, as well as legacy UNIX utilities such as $1 \mathrm{~s}, \mathrm{cp}$, and rm. PVFS also allows read-only memory mapping of files through the mmap system call interface. Interfacing to the UNIX I/O functions is accomplished by loading a Linux kernel module that hooks into the appropriate place in the Linux VFS layer without having to recompile the kernel and/or reboot the machine. Once mounted, the PVFS file system can be traversed and accessed with existing binaries just as any other file system. Many network file systems like NFS have weaker consistency guarantees on file system data and meta-data, since they are primarily targeted at workloads where it is not common to have many processes accessing the same files or directories from many nodes simultaneously. PVFS, on the other hand, cannot afford to have such weaker file system semantics guarantees because it is primarily targeted at workloads that exhibit read-write data sharing. Therefore, PVFS (at this stage) does not cache file data and meta-data in the Linux page cache; in other words, all file system accesses have to incur a network transaction.

\subsection{Design of the PVFS Kernel Module}

When the PVFS kernel module is loaded, it registers itself with the Linux VFS layer. After the PVFS file system is mounted, subsequent file system calls on the PVFS volume are dispatched by the VFS layer to the module. The module uses a device file to communicate the request to a user level daemon (pvfsd) which satisfies the requests by talking to the MGR and/or the IOD servers. This design is conceptually similar to the Coda design [12] and is shown in Figure 2. We briefly go over a simplified algorithm for the read, write control paths and the daemon here.

- Read

1. Enqueue a read request to the character device queue.

2. Wake up the daemon (pvfsd) if necessary. 


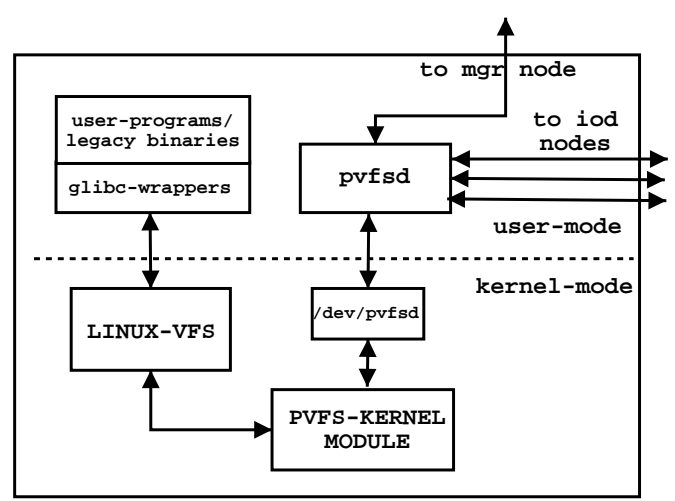

Figure 2. System architecture

3. Wait until interrupted by a signal or when operation is complete. Completion is indicated by the daemon once the data is copied from its address space to the kernel buffers.

4. If operation is complete, copy the data from the kernel buffers to the user space virtual addresses; else return appropriate error code.

- Write

1. Enqueue a write request to the character device queue. This step also involves copying the data from the program's user space virtual addresses to the kernel's temporary buffers.

2. Wake up the daemon (pvfsd) if necessary.

3. Wait until interrupted by a signal or until the daemon writes back the virtual address to which the data to be written needs to be copied.

4. Copy the data from the kernel buffers to the daemon's virtual address.

5. Enqueue another write request to the character device queue. Wait until the operation is complete or for a signal to terminate the operation.

6. Return appropriate error codes or operation success

- User-Space Daemon

1. Block on the device queue waiting for a request to appear.

2. Read the request from the character device queue. Ascertain the operation type.

3. If it is a write request that was enqueued in Step 1 , allocate a virtual address region large enough to accommodate the write, and enqueue an acknowledgment into the device queue.

4. If it is a write request that was enqueued in Step 5 or any other operation (meta-data/read), stage the appropriate operation, and enqueue the results of the operation as an acknowledgment into the device queue, waking up the blocked process.

On the one hand, a user space daemon-based approach such as the above lends well to better code reuse, easier debugging, and simplicity. On the other hand, we observed that it does not perform well for many read/write-intensive workloads because of context switching and buffer copying overheads as elaborated upon in Section 3.

\section{Improving Performance}

The chief performance bottlenecks that we address in the subsequent sections are

- increased buffer copies and context switching overheads,

- resource/socket utilization overheads, and

- lack of aggregation in directory read operations.

\subsection{Reducing Buffer Copy and Context Switch- ing Overheads}

As illustrated earlier, a typical read/write operation incurs at least two buffer copies before the data gets into/from the applications address space (one copy incurred when transferring from/to the daemon's address space to/from temporary kernel buffers, one copy incurred when copying from/to kernel buffers to/from the user program's address space, not including the daemon's read/write from/to the kernel socket buffers when communicating with the IOD and MGR servers). Additionally, the write operation incurs an extra latency because of the extra step involved in waiting for a suitably large virtual address from the daemon's address space. Moreover, each of these operations involves at least two context switches before the file data is written out to the file system or read into the application's address space. For all these reasons, the above design turned out to be inappropriate for a high-performance parallel file system.

Beginning with Linux kernel version 2.4, two APIs, kernel_thread (which was essentially a front end for the clone system call) and daemonize are exported to kernel modules to create kernel threads with no user space context associated with them. This approach allows modules to create active entities that execute completely inside the kernel. Since they have no associated user space component, context switching overheads are less. In addition, since they run inside the kernel address space, they can use the temporary kernel buffers directly to stage reads or writes without any extra copying. Moreover, in recent times there have been trends to incorporate many traditional user space services into the kernel, most notably Web servers such as TUX [9], for the sake of performance. Therefore, this mechanism can be used to avoid buffer copying, system call, and context switching overheads. Another consequence of executing in kernel space is the fact that we can remove the character device interface altogether and have the daemon operate on the queues directly. With the new mechanism, the read/write control paths and the daemon's pseudo-code are briefly described below:

- Read

1. Enqueue a read request to the queue.

2. Wake up the kernel space daemon (kpvfsd) if necessary. 
3. Wait until interrupted by a signal or when operation is complete. Completion of operation is indicated by the daemon, when the data transfer to the kernel buffers finishes.

4. If operation is complete, copy the data from the kernel buffers to the user space virtual addresses; else return appropriate error code.

- Write

1. Enqueue a write request to the queue. This step also involves copying the data from the program's user space virtual addresses to the kernel's temporary buffers.

2. Wake up the kernel space daemon (kpvfsd) if necessary.

3. Wait until interrupted by a signal or when operation is complete. Completion of operation is indicated by the daemon, when the data has been successfully transferred to the I/O nodes.

4. Return appropriate error codes or operation success.

- Kernel-Space Daemon

1. Block on the queue waiting for a request to appear.

2. Read the request directly from queue. Ascertain the operation type.

3. Stage the read/write operation directly to/from the kernel buffers.

4. Enqueue the results of the operation as an acknowledgment into the queue, waking up the blocked process.

Note that in the above algorithm, we have removed the additional step for writes that was needed in the user space daemon case, and thus we expect to see higher benefits for writes than for reads. This method is shown in Figure 3.

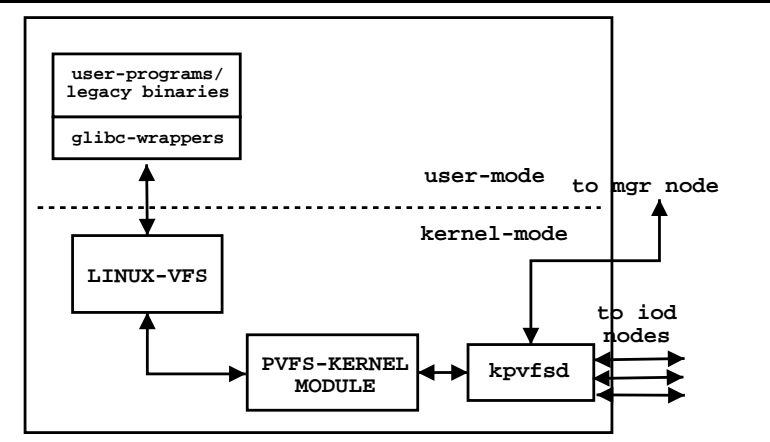

Figure 3. Modified system architecture

\subsection{Resource Utilization Management}

As stated earlier, the client-side daemon uses TCP sockets to connect and exchange information with the IOD servers. The previous implementation managed connections to the IOD servers at the granularity of a file i.e., it kept as many socket connections per file as there are number of IOD servers over which the file is striped. While such an approach is simple, it suffers from serious scalability and performance issues when there are many simultaneous open files and IOD servers. It is a scalability issue for two reasons, namely, the limited number of open file descriptors that is usually permitted for a process, and the poor scalability of the select system call with a large number of file descriptors [3] on the servers. It is a performance issue because of the overhead involved in setting up and tearing down connections to the servers for every new file that is accessed. The overhead is even greater for clients that access the file system through the PVFS library, since the number of file descriptors is potentially increased by a factor proportional to the number of processes that are executing on the node.

The new implementation manages connections at a node granularity; in other words, we keep as many connections as there are IOD servers, and reuse the connections for all files. This approach has not only improved performance for workloads that manipulate a large number of files, but has also improved the scalability when there are many simultaneously open files and IOD servers. In Section 4, we present experimental results from a synthetic benchmark that times the untar of the Linux kernel source tree to illustrate the benefits of the IOD connection management.

\subsection{Aggregation of Directory Read Operations}

A final improvement in performance that we discuss in this paper, is the aggregation of directory read operations on a PVFS file system. As stated earlier, since PVFS is primarily targeted at workloads where it is normal to have many clients accessing the same files and directories concurrently, it does not cache directory entries on the client machine's directory entry cache. While such a design greatly simplifies consistency semantics issues, it comes at the cost of performance. Aggregation in directory read operations is hindered by the fact that the number of entries in a directory is not known without a network transaction to the MGR server. Hence, glibc wrappers for the getdents system call make estimates based on the size reported by a stat on the directory, and repeatedly make the system call until all the entries have been exhausted or there is an error. Therefore, the previous implementation makes a network call for obtaining every directory entry that was extremely slow. Since the memory for copying the directory entry is allocated by $\mathrm{glibc}$ and the sizes of directory entries as reported by the kernel is dependent on what struct dirent definition is being used by the system, the VFS layer passes an opaque (opaque to the file system) pointer to the directory entry and an opaque call back function (filldir_t) to the underlying file system which returns an error when space provided by $g$ libc is exhausted. Thus, the underlying file system must repeatedly call the opaque function until it returns an error. The new implementation makes a request to the MGR server to fetch a fixed number of directory entries at a time (currently 64) to amortize the network transfer costs, and repeatedly invokes the filldir_t function until it returns an error. In Section 4, we illustrate the performance benefits of this approach by timing a recursive listing of a PVFS 
file system directory containing the Linux kernel source tree.

\section{Experimental Results}

All these experiments were conducted on a 16-node Intel Pentium-III based cluster running Linux kernel 2.4.18 using both the fast Ethernet-based network and a Myrinetbased network. The platform was configured to run the MGR server on one of the nodes (on port 3000) and two instances of the IOD servers on each of the nodes (one each for the Myrinet and the Ethernet interfaces on ports 7000 and 7001). Only one IOD server was used per node for each test run. In effect, we created two separate 16 IOD server PVFS file systems on the platform.

\subsection{Aggregate Bandwidth Tests}

Our first test program is a parallel MPI program that determines the aggregate read/write bandwidths for varying block sizes, iteration count, and number of clients (pvfs_test.c from the PVFS distribution). Each process opens a new PVFS file that is common to all processes, concurrently writes data blocks to disjoint regions of the file, closes the file, reopens the file, reads the same data blocks back from the file, and then closes the file. The tasks of the parallel application synchronize before and after each I/O operation. Times for the read/write operations on each node are recorded over five trial runs and the maximum averaged time over all the tasks is used to compute the bandwidth achieved. In the first experiment, we varied the block sizes and ran the experiment by using the POSIX I/O interface using both the user space and kernel space daemons for a fixed number of clients. The graphs in Figures 4 and 5 plot the file system read and write bandwidth as a function of the block size for the POSIX interface when using Myrinet for 8 and 16 clients. Figures 6 and 7 plot the same for fast Ethernet. While there is a clear benefit of using a kernel-space approach for both the networks, the benefits are more apparent for a faster network like Myrinet. In the case of Myrinet, we achieve around 20\% improvement in read band width for 8 clients; the improvement falls off slightly when using 16 clients. Writes with Myrinet give nearly $40 \%$ improvement, which again drops with increased number of clients. The performance improvements for a smaller number of clients can be attributed to the removal of an additional step from the write process. For a larger number of clients, the difference gets smaller because the dominating bottlenecks are possibly due to the servers. We achieved nearly $500 \mathrm{MB} / \mathrm{sec}$ read bandwidth and $550 \mathrm{MB} / \mathrm{sec}$ write bandwidth using Myrinet. The benefits when using a fast Ethernet-based network are not significant, however, indicating that the network transfer speeds are a bottleneck. The benefits accrued as a result of reduced context switches and copy overheads start to show up only at intermediate ranges of block sizes. In this case, we achieve anywhere from 2 to $10 \%$ improvement in bandwidth, and larger improvements are observed for writes only (for the same reason as above). We achieve nearly $150 \mathrm{MB} / \mathrm{sec} \mathrm{read}$ and $160 \mathrm{MB} / \mathrm{sec}$ write bandwidth using fast Ethernet.

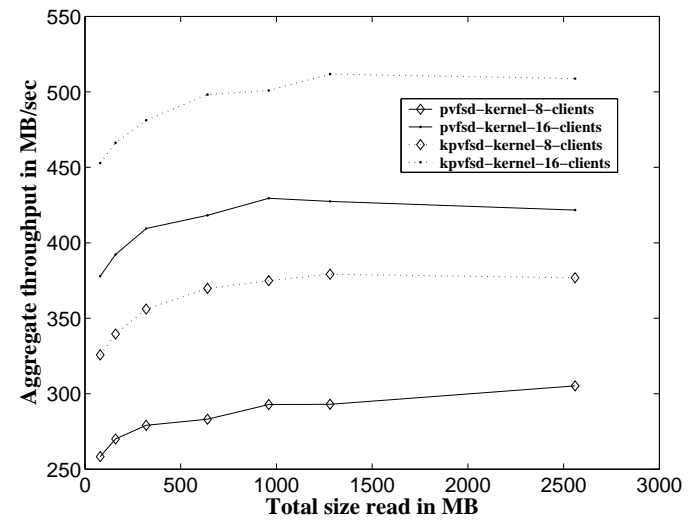

Figure 4. Myrinet: aggregate read bandwidth

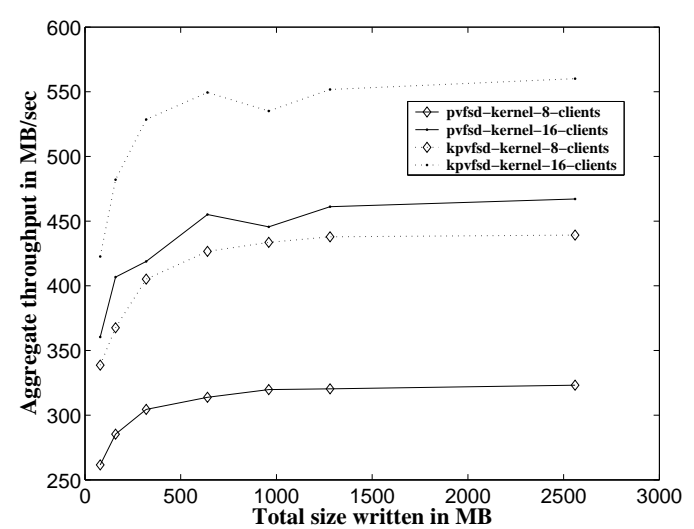

Figure 5. Myrinet: aggregate write bandwidth

\subsection{Bonnie++}

Our second benchmark is the Bonnie++ benchmark, which performs a series of tests on the file system and reports a number of metrics. The first six tests simulate file system activity that has been observed to be a bottleneck in I/O-intensive applications. Bonnie++ performs a series of tests on a file of a known size and reports the number of kilobytes processed per second and the percentage of CPU used for each of the test. The second set of six tests simulate operations such as create, stat, and unlink, which are observed to be common bottlenecks on proxy web cache servers, news servers and email servers. The reader is referred to [7] for more information on Bonnie++.

4.2.1. File $\mathrm{I} / \mathrm{O}$ Tests The file $\mathrm{I} / \mathrm{O}$ tests that are reported by Bonnie++ are of three kinds, namely, sequential writes, sequential reads, and random seeks. The sequential writes are done either per character (using putc) or per block (using write) or are rewritten (using read, overwrite, write). The sequential reads are done either per character (using get c) or per block (using read). The random seek test forks a certain number of processes, each of which does a total of 8,000 lseek's to random loca- 


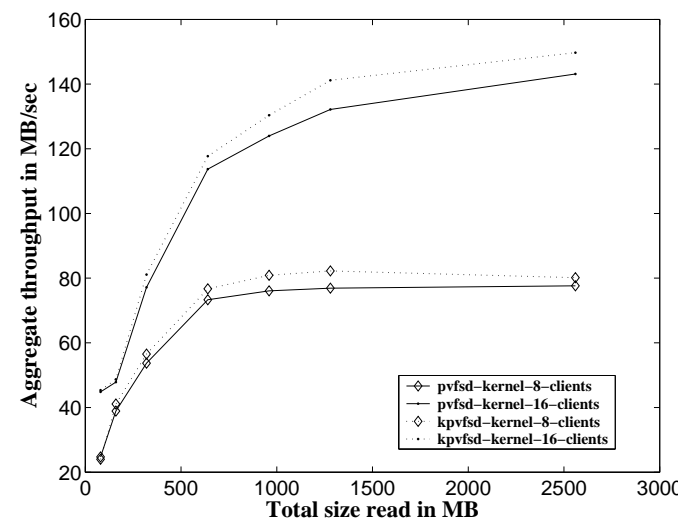

Figure 6. Fast Ethernet: aggregate read bandwidth

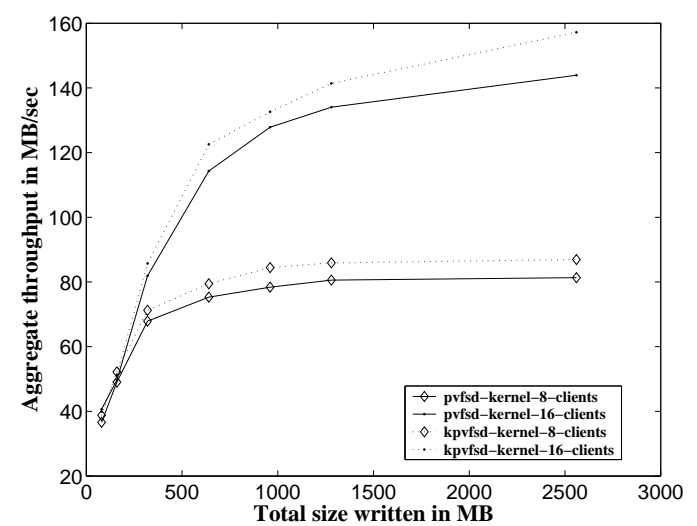

Figure 7. Fast Ethernet: aggregate write bandwidth

tions in the file. After the seek, the block is read, dirtied, and written back in $10 \%$ of the cases. In all these experiments, Bonnie++ was configured to use a chunk size of $16 \mathrm{~KB}$, and we varied the file size from $32 \mathrm{MB}$ to 512 MB. Since PVFS does not cache file data on the client machine, none of these tests - with the exception of the put c test - should be affected significantly by any buffering/caching effects, since data is always transferred over the network. Hence, performance remains unaffected not only with file size, but also with memory size which is required as a parameter to Bonnie++ (unless it is set to such an extremely low value that Bonnie++ will start thrashing). Hence, we show representative results for selected file sizes. Throughput metrics for the read/write tests in Bonnie++ are in $\mathrm{KB} / \mathrm{sec}$, while seek throughputs are measured as the rate per second. These experiments were conducted for both the kernel space and user space daemon implementation on both a Myrinet-based and fast Ethernet-based network. We show the percentage performance improvements obtained with fast Ethernet in Figure 8 and Myrinet in Figure 9. We expected that the percentage improvement with a kernel-space approach would be felt only when the reads and writes were done using the blocked I/O method and not when using a per character-based method, since the main savings of this approach are the reduction in copying and context switching overheads, which are not too dominant with a per character-based approach. We see from the graphs, that while rewrites and blocked reads performed exceptionally well with a kernel-space approach, where we achieve nearly $90 \%$ improvement using fast Ethernet and around 200\% improvement using Myrinet, we do not see a significant improvement in the performance of blocked writes, an anomalous behavior that we cannot yet explain. We also see a significant improvement in the random seek test of nearly $60 \%$ in both the networks. (As expected, the per character-based techniques do not achieve any significant benefits with this approach). The increase in performance with this approach does come with a price. Figure 10 show the absolute percentage CPU utilization for the same tests for the Myrinet experiment. We see that in almost all the cases, we incur a slightly higher CPU utilization overhead, which we surmise is due to the fact that in most cases the transfer is being performed over less time.

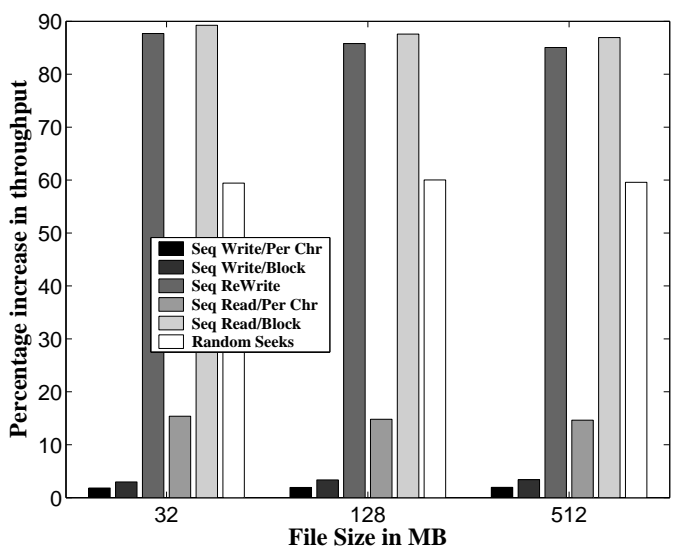

Figure 8. Fast Ethernet: Percentage increase in throughput for Bonnie++

4.2.2. File Creation Tests We used two file creation tests from Bonnie++: sequential test, where a random number of alpha-numeric characters follow a seven-digit number to construct the file name, and a random test where the random characters precede the seven-digit number. The sequential tests create the files in increasing numeric order, stat them in the readdir order, and unlink them in the same order. The random tests create the files in random order (appears random because of the alphanumeric characters in front of the seven-digit number in the filename), stat them in random order, and unlink all the files in random order. In these tests, it is also possible to specify the maximum size and the minimum size of the files that are created, in which case the files are written to at creation and read back after the stat. We report this experiments run for the Myrinet network only, since the results are not significantly different for the fast Ethernet-based network. The output from this test is the op- 


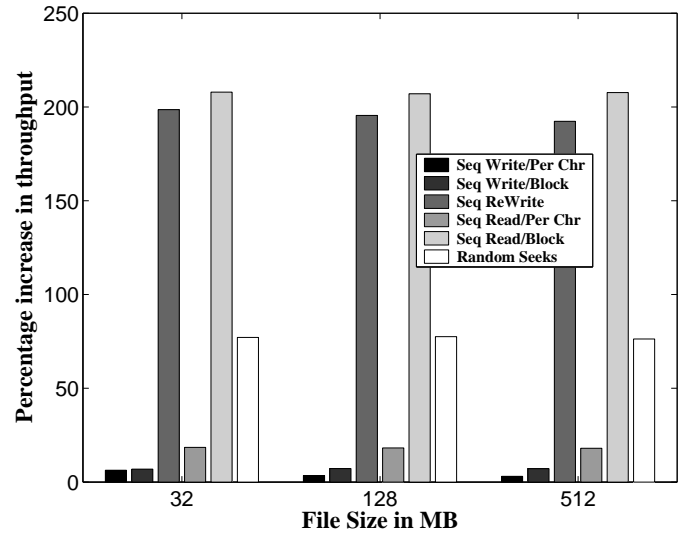

Figure 9. Myrinet: Percentage increase in throughput for Bonnie++

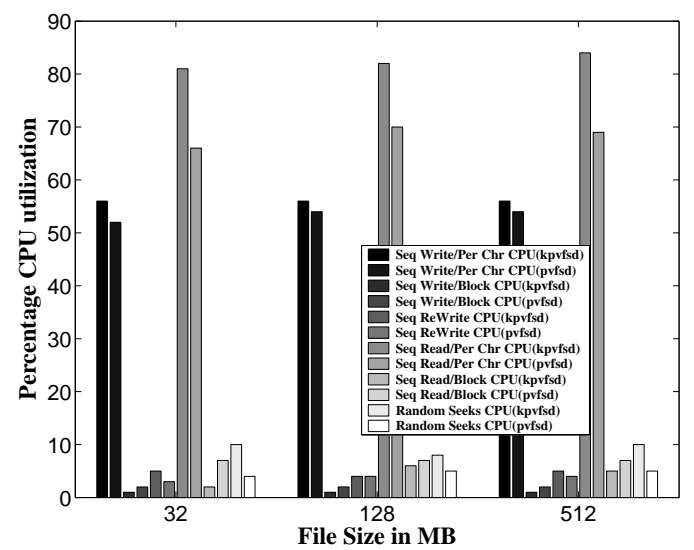

Figure 10. Myrinet: CPU utilization for Bonnie++

eration rate measured per second and is parameterized by the number of files, which we varied from 1024 to 4096. Since these tests don't really transfer large amounts of data, the benefits of using a kernel space approach are not expected to be significant, and Figure 11 reinforces the belief. Further, we see particularly low transaction rates for create operations and small file operations; this will be further investigated in the next section. There is no significant change in the CPU utilization for this workload.

\subsection{IOD Connection Management}

While the scalability benefits of managing connections to the IOD server at a node granularity is apparent, we wanted to quantify the performance benefits of this approach. Table 1 lists the time taken with and without the connection management for untarring the Linux 2.5.39 kernel source tree on a PVFS volume for both the user space and kernel space daemon-based approaches. From Table 1 , we see that this strategy did improve performance but

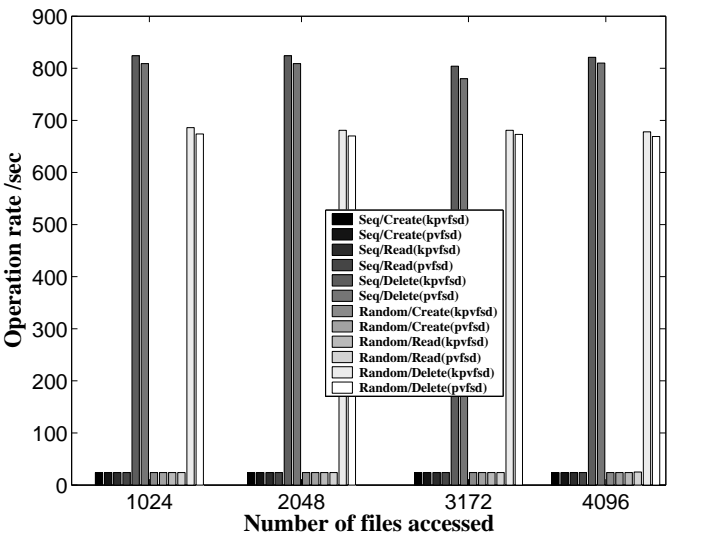

Figure 11. Myrinet: small file operation throughput

\begin{tabular}{|l|r|r|r|r|}
\hline & $\begin{array}{r}\text { pvfsd } \\
\text { no-mgmt. }\end{array}$ & $\begin{array}{r}\text { pvfsd } \\
\text { mgmt. }\end{array}$ & $\begin{array}{r}\text { kpvfsd } \\
\text { no-mgmt. }\end{array}$ & $\begin{array}{r}\text { kpvfsd } \\
\text { mgmt. }\end{array}$ \\
\hline Fast Ethernet & $1067.5 \mathrm{sec}$ & $1017.96 \mathrm{sec}$ & $1045.55 \mathrm{sec}$ & $1008.50 \mathrm{sec}$ \\
\hline Myrinet & $959.4 \mathrm{sec}$ & $903.41 \mathrm{sec}$ & $933.64 \mathrm{sec}$ & $885.90 \mathrm{sec}$ \\
\hline
\end{tabular}

not significantly (nearly 3\% for fast Ethernet and 5\% for Myrinet). In order to investigate the reasons for the insignificant performance improvements for this workload, we instrumented the kernel module to count the number of requests of each type and compute the average time spent by the requesting process for its request to be serviced. These statistics are made accessible to user-space through the proc file system. Table 2 lists the time spent (in $\mu$ seconds) by a process, while waiting for its request to be serviced by the daemon (Myrinet-based network) for this workload. This general purpose file system workload is characterized by meta-data intensive operations and small file data transfers. As the table illustrates, the meta-data operations, and in particular, the create operation dominates over the rest. A create operation is directed to the single MGR server, which then checks permissions, and fans out requests to the appropriate IOD servers directing them to open this file. Once it gets acknowledgments from all the IOD servers, it sends back an acknowledgment to the client, which includes amongst other things, the locations of the IOD servers and physical distribution of file data. Thus, the poor performance of meta-data operations can be attributed to the following reasons; serialization imposed by the single MGR server, communication overhead with the IOD servers, and the lack of a client-side meta-data cache. Moreover, PVFS was designed with a goal of providing high-performance parallel I/O for large data transfers, and it has not been well-tuned for small file operations (The 2.5.39 kernel source tree has an average file size of around $10 \mathrm{~KB}$ ) or for meta-data intensive operations.

\subsection{Aggregation of Directory Read Operations}

The performance improvements obtained by aggregating directory read operations are shown in Table 3. 


\begin{tabular}{|l|r|r|r|r|r|}
\hline $\begin{array}{l}\text { Upcall } \\
\text { type }\end{array}$ & count & $\begin{array}{r}\text { pvfsd } \\
\text { no-single }\end{array}$ & $\begin{array}{c}\text { pvfsd } \\
\text { single }\end{array}$ & $\begin{array}{c}\text { kpvfsd } \\
\text { no-single }\end{array}$ & $\begin{array}{c}\text { kpvfsd } \\
\text { single }\end{array}$ \\
\hline create & 15695 & 41931 & 38605 & 41411 & 38493 \\
\hline setmeta & 17764 & 3052 & 3025 & 2975 & 2970 \\
\hline write & 30640 & 2745 & 2717 & 2674 & 2637 \\
\hline read & 1140 & 2277 & 3180 & 2124 & 2185 \\
\hline mkdir & 2070 & 1693 & 1681 & 1652 & 1666 \\
\hline getmeta & 152607 & 978 & 912 & 944 & 878 \\
\hline lookup & 35531 & 374 & 367 & 335 & 330 \\
\hline \hline total & 255447 & 3778 & 3532 & 3705 & 3482 \\
\hline
\end{tabular}

Table 2. Myrinet: Average time spent waiting in queue (in $\mu \mathrm{sec}$ )

\begin{tabular}{|l|l|l|l|l|}
\hline & $\begin{array}{l}\text { pvfsd- } \\
\text { unpatched }\end{array}$ & $\begin{array}{l}\text { kpvfsd- } \\
\text { unpatched }\end{array}$ & $\begin{array}{l}\text { pvfsd- } \\
\text { patched }\end{array}$ & $\begin{array}{l}\text { kpvfsd- } \\
\text { patched }\end{array}$ \\
\hline Fast Ethernet & $881.32 \mathrm{sec}$ & $879.78 \mathrm{sec}$ & $177.18 \mathrm{sec}$ & $173.22 \mathrm{sec}$ \\
\hline Myrinet & $878.69 \mathrm{sec}$ & $877.19 \mathrm{sec}$ & $158.77 \mathrm{sec}$ & $153.81 \mathrm{sec}$ \\
\hline
\end{tabular}

Table 3. Recursive directory listing

It lists the time taken to recursively list a PVFS directory, which contains the Linux 2.5.39 kernel source code for both a fast Ethernet-based network and a Myrinet-based network. The Linux kernel source tree has around 15,000 files spread over 2,070 directories. The command we used to time this experiment was, time ls -aR/mnt/pvfs/linux-2.5.39/ $>/ \mathrm{dev} / \mathrm{null}$. As expected, directory aggregation increases performance by almost a factor of 5 . The kernel space approach, however, does not significantly improve the performance (as compared to a user space approach) in both the networks, since the volume of data transferred is not very large. We note here that, this is still much slower than for a network file system with client-side caching of directory entries. For instance, the time taken to recursively list the same kernel source tree on NFS on the same platform is nearly 10 seconds. Hence, a lot of performance tuning still remains to be done.

\section{Concluding Remarks and Future Work}

PVFS is an actively supported, high-performance, robust, and usable parallel file system for Linux-based commodity clusters. It supports a number of different APIs, most notably the UNIX/POSIX I/O API and the MPIIO API, which allow legacy binaries as well as highperformance scientific applications to access PVFS file systems. In this paper, we have addressed several performance and scalability problems of the PVFS kernel module that allows applications to access PVFS file system with the standard UNIX I/O interfaces. Applications which read and write large amounts of data will be benefited due to the reduction of buffer-copies and context switches. Managing socket connections to the IOD servers benefits applications which manipulate a large number of files and aggregation of directory operations benefits applications which access and manipulate directory structures.

Considerable room for improvement and tuning remains. While PVFS has been optimized for applications that demand high file system bandwidths, it is still largely untuned for small file operations and meta-data intensive operations, which are important for many gen- eral purpose applications like mail and news servers. Work is already underway on PVFS2 that eliminates the dependence on a single meta-data server to improve performance of small file operations and meta-data intensive workloads. Performance could be increased further by having a pool of client-side kernel threads (possibly limited by the number of CPUs, and having each thread bound to a $\mathrm{CPU}$ ) that services requests from per $\mathrm{CPU}$ request queues or by employing an event driven non-blocking approach to servicing requests out of order from the request queue.

\section{References}

[1] Intel Scalable Systems Division, Paragon system user's guide, 1995.

[2] IEEE/ANSI Std. 1003.1 Portable Operating System Interface (POSIX)-Part 1: System Application Program Interface (API) [C Language]., 1996.

[3] G. Banga, J. C. Mogul, and P. Druschel. A Scalable and Explicit Event Delivery Mechanism for UNIX. In Proceedings of the USENIX Annual Technical Conference, pages 253265, 1999.

[4] N. J. Boden, D. Cohen, R. E. Felderman, A. E. Kulawik, C. L. Seitz, J. N. Seizovic, and W. Su. Myrinet: A Gigabitper-Second Local Area Network. IEEE Micro, 15(1):29-36, 1995.

[5] P. H. Carns, W. B. Ligon III, R. B. Ross, and R. Thakur. PVFS: A Parallel File System for Linux Clusters. In Proceedings of the 4th Annual Linux Showcase and Conference, pages 317-327, Atlanta, GA, 2000.

[6] A. Ching, A. Choudhary, W. Liao, R. B. Ross, and W. Gropp. Noncontiguous I/O through PVFS. In Proceedings of the IEEE International Conference on Cluster Computing, 2002.

[7] R. Coker. Bonnie++ file-system benchmark. On the WWW at http: //www. coker.com.au/bonnie++/.

[8] M. P. I. Forum. MPI-2: Extensions to the Message-Passing Interface, 1997. http://www.mpi-forum.org/docs.

[9] I. Molnar. Tux Web Server 1.0. http://people.redhat.com/mingo/TUX-PATCHES/.

[10] S. A. Moyer and V. S. Sunderam. PIOUS: A scalable parallel I/O system for distributed computing environments. In Proceedings of the Scalable High-Performance Computing Conference, pages 71-78, 1994.

[11] N. Nieuwejaar and D. Kotz. The Galley parallel file system. In Proceedings of the 10th ACM International Conference on Supercomputing, pages 374-381, Philadelphia, PA, 1996. ACM Press.

[12] M. Satyanarayanan, J. J. Kistler, P. Kumar, M. E. Okasaki, E. H. Siegel, and D. C. Steere. Coda: A Highly Available File System for a Distributed Workstation Environment. IEEE Transactions on Computers, 39(4):447-459, 1990.

[13] F. Schmuck and R. Haskin. GPFS: A shared-disk file system for large computing clusters. In Proceedings of the First Conference on File and Storage Technologies (FAST), 2002.

[14] R. Thakur, W. Gropp, and E. Lusk. An Abstract-Device Interface for Implementing Portable Parallel-I/O Interfaces. In Proceedings of the Sixth Symposium on the Frontiers of Massively Parallel Computation, pages 180-187, 1996.

[15] R. Thakur, E. Lusk, and W. Gropp. Users Guide for ROMIO: A High-Performance, Portable MPI-IO Implementation. Technical Report ANL/MCS-TM-234, Argonne National Labs, 1997. 